\title{
In vivo Blood-brain Barrier Permeability Assays \\ Using Clostridium perfringens Epsilon Toxin
}

Michael R. Mazzucco, Timothy Vartanain and Jennifer R. Linden*

Brain and Mind Institute, Weill Cornell Medical College, New York City, New York, 10065, USA

*For correspondence: jel2049@med.cornell.edu

[Abstract] In order for the brain to function properly, a carefully orchestrated homeostasis must be maintained. To help regulate this delicate balance, the brain has developed a highly selective bloodbrain barrier (BBB). Under normal conditions, the BBB excludes harmful blood-borne material from the brain parenchyma. However, numerous neuropathological conditions can disrupt this barrier, causing BBB permeability and subsequent CNS dysfunction. Understanding the mechanisms involved in BBB permeability are essential to elucidating the pathology of various neurological disorders as well as identifying methods for drug delivery to the CNS. Here, we describe several in vivo methods to measure BBB permeability in mice using an array of diverse sized tracers including exogenous 376 Da fluorescein salt, $66.5 \mathrm{kDa}$ bovine serum albumin, and $70 \mathrm{kDa}$ dextran as well as endogenous $160 \mathrm{kDa}$ mouse IgG. When administered intravenously, these substances are excluded from a healthy brain by the BBB. However, BBB dysfunction can allow entry of these tracers into the brain and this accumulation can be measured using spectrophotometry, fluorescent microscopy, and immunohistochemistry. We also describe a method to induce BBB permeability using Clostridium perfringens epsilon toxin. Finally, we include a short discussion about the advantages and disadvantages of each method and their appropriate downstream applications.

Keywords: In vivo, Blood-brain barrier, Permeability, Murine model, Clostridium perfringens, Epsilon toxin

[Background] To maintain the intricate homeostasis required for proper brain function, the central nervous system (CNS) has developed a selective barrier that stringently regulates movement of bloodborne material into the brain. This is called the blood-brain barrier (BBB). This barrier is generated by highly specialized brain endothelial cells (BEC) whose unique properties are influenced by the close contact of CNS pericytes and astrocytes (Balabanov and Dore-Duffy, 1998; Engelhardt, 2003; Abbott et al., 2006; Abbott and Friedman, 2012; Alvarez et al., 2013). This increased barrier activity is achieved using two main mechanisms in BEC. The first is reduced paracellular permeability via formation of tight junctions at cell-to-cell contacts and the second is decreased transcellular permeability through reduced pinocytic activity (Gloor et al., 2001; Wolburg and Lippoldt, 2002; Preston et al., 2014; Tietz and Engelhardt 2015; De Bock et al., 2016). An increase in BBB permeability can have devastating neuropathological outcomes and can result in death. Because of the destructive consequences that BBB permeability can have on normal brain function, it is important to establish methods that reliably measure BBB permeability to help identify both the causes of and treatments for BBB dysfunction. 
To measure BBB permeability in vivo in mice, animals are typically injected intravenously (IV) with a tracer or dye normally excluded from the brain by an intact BBB. The most commonly used tracer is Evans Blue (Saunders et al., 2015). However, it has been argued that Evans Blue has significant limitations as a tracer for BBB permeability assays and has been extensively reviewed in Saunders et al. (2015). Because Evan Blue binds to plasma albumin, extraversion of Evans Blue into the CNS is reasoned to be a measurement of albumin permeability. However, it has been demonstrated that Evans Blue binds to several other plasma proteins and may even be present as a free dye, suggesting that Evans Blue is an unsuitable tracer for BBB permeability. To help reduce the amount of uncertainty of accessing BBB permeability with Evan's Blue, we sought to identify tracers that were better characterized and would therefore allow us to better define the specific aspects involved in BBB dysfunction. For our purposes, we wished to identify the size of molecules a damaged BBB had become permeable to, indicating if certain blood proteins could cross the BBB. Extravasation of certain bloodborne proteins such as complement, fibrinogen, and immunoglobulins may have lasting pathological effects on the CNS even after a permeable BBB has been repaired. Based on these criteria, we selected a panel of easily detectable tracers including exogenous 376Da fluorescein salt (FITC-Na), $66.5 \mathrm{kDa}$ Alexa fluor 594 conjugated bovine serum albumin (BSA-594), and $70 \mathrm{kDa}$ fluorescein isothiocyanate conjugated dextran (FITC-dextran); and the endogenous $155 \mathrm{kDa}$ tracer, mouse IgG.

In this paper, we outline procedures on how to use FITC-Na, BSA-594, -FITC-dextran, and endogenous IgG as tracers for BBB permeability and their acceptable downstream applications. In addition, we also describe a method to induce BBB permeability in mice using Clostridium perfringens epsilon toxin (ETX) (Linden et al., 2019). ETX-induced BBB permeability opens the BBB to all the tracers described here, specifically through caveolae dependent-transcytosis using both receptor-mediated and fluid-phase mechanisms (Linden et al., 2019). This panel allows us to access permeability to substances with varied molecular sizes and well as different molecular characteristics. FITC-Na can be used as a measure of solute and ion permeability, while BSA, $70 \mathrm{kDa}$ dextran, and IgG can be used as measures of protein permeability (Nag, 2003). Influx of specific markers may also help identify what paracellular or transcellular mechanisms are involved in BBB dysfunction. For example, albumin transport is mediated via caveolae dependent-transcytosis (Schnitzer et al., 1994; Schubert et al., 2001; Frank et al., 2003). However, specific mechanisms of BBB permeability need to be confirmed using additional experimental methods. We strongly suggest ultrastructure examination via electron microscopy to determine if permeability is a result of increased paracellular permeability via tight junction dysfunction or increased transcellular permeability because of elevated endocytic activity. We also describe the best methods to use to evaluate extravasation for each tracer, as some techniques are not compatible with certain tracers, and can result in significant reduction of signal (Buxton, 1978; Hoffmann et al., 2011, Saunders et al., 2015). Finally, we also describe some of the advantages and disadvantages for each of the methods and tracer combinations (Figure 1). These methods may be used to measure BBB permeability in numerous disease models, transgenic mouse models, and CNS drug delivery evaluations. 


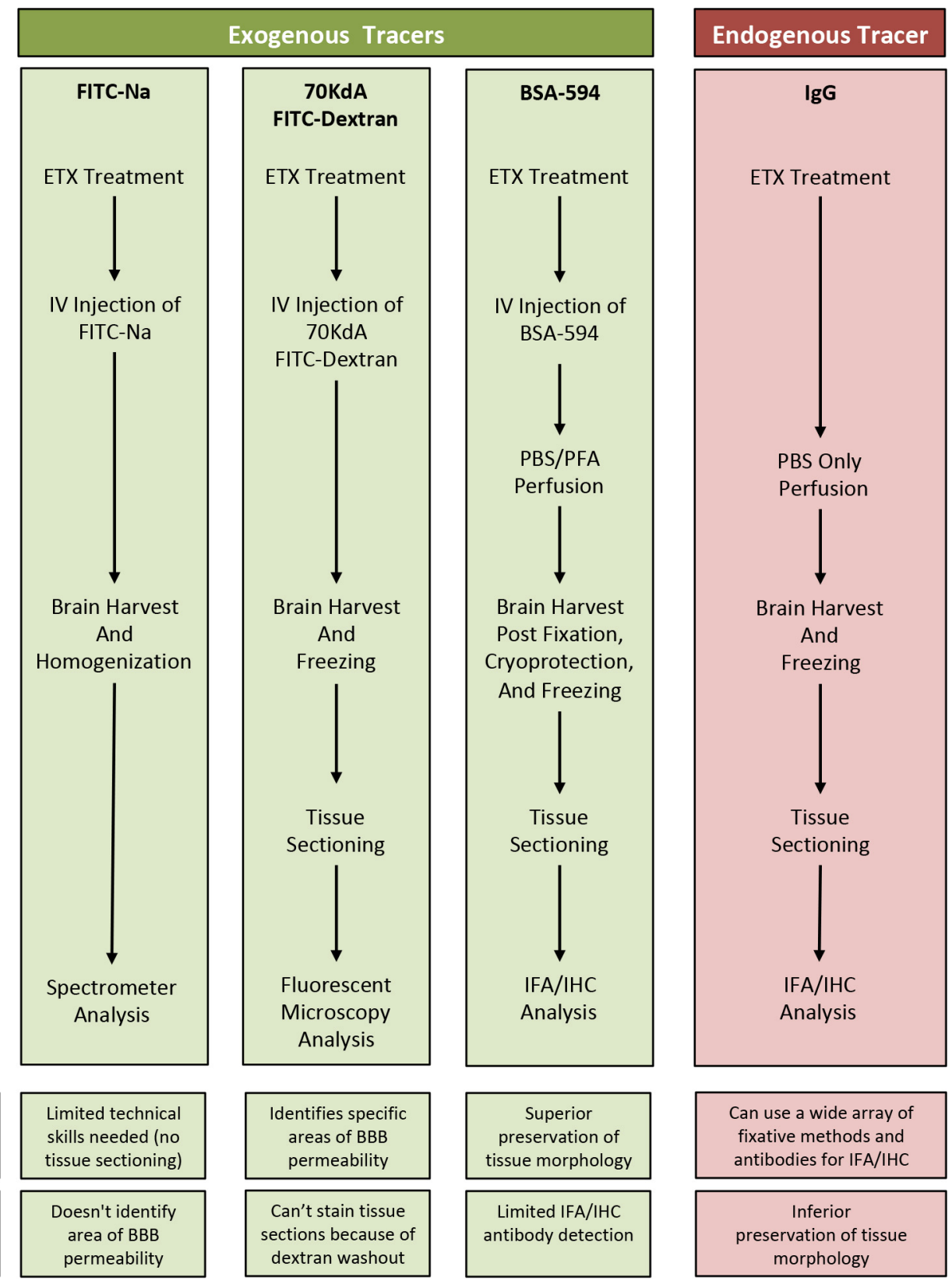

Figure 1. Overview of specific tracer and post-treatment workflow highlighting advantages (PRO) and disadvantages (CON) of each method

\section{Materials and Reagents}

1. Clear nail polish (any brand)

2. Microscope slide storage box (any brand)

3. Razor blades (any brand)

4. $2 \mathrm{~mm}$ glass beads (Sigma, catalog number: Z273627)

5. Microcentrifuge tube (Sigma, catalog number: CLS320)

6. Kimwipes 
7. Insulin Syringes, 29 Gauge, $0.5 \mathrm{ml}$ volume, $12.7 \mathrm{~mm}$ needle length (BD, catalog number: 324703)

8. Intermediate Size Cryomolds (Tissue-Tek, catalog number: AGG4582)

9. $22 \times 50 \mathrm{~mm}$ Rectangular \#11/2 Cover Glass (Corning, catalog number: $2980-225$ )

10. Superfrost Plus Microscope Slides (Fisherbrand, catalog number: 12-550-15)

11. C57BL/6J mice, female, 8 weeks old (The Jackson Laboratory, catalog number: 000664)

12. $70 \%$ Ethanol

13. Epsilon protoxin (Biodefense and Emerging Infections Research Resources Repository, catalog number: NR-856), store at $-80^{\circ} \mathrm{C}$

14. Immobilized TPCK-Trypsin resin (Thermo Scientific, catalog number: 20230), store at $4{ }^{\circ} \mathrm{C}$

15. Sodium phosphate monobasic dehydrate (Sigma, catalog number: 71505)

16. Sterile Saline Solution: $0.9 \%$ sodium chloride solution, $10 \mathrm{ml}$ vial, Single-dose, for use as sterile diluent (Hospia, Inc.), store at room temperature

17. FITC-Na (Fluorescein sodium salt) (Sigma, catalog number: F6377), store at room temperature protected from light

18. $70 \mathrm{kDa}$ FITC-dextran (Fluorescein isothiocyanate-dextran with average molecular weight of $70,000 \mathrm{Da}$ ) (Sigma, catalog number: 46945 ), store at $4{ }^{\circ} \mathrm{C}$ protected from light

19. BSA-594 (Albumin from Bovine Serum), Alexa Fluor 594 conjugated (Thermo Fisher Scientific, catalog number: $\mathrm{A} 13101)$, store at $4{ }^{\circ} \mathrm{C}$ protected from light

20. PBS (Invitrogen, catalog number: AM9625), store at room temperature

21. Xylazine and ketamine cocktail, obtained from institutional Research and Animal Resource Center or equivalent

22. Tissue-Tek ${ }^{\circledast}$ OCT Compound (Sakura Finetek, catalog number: 4583), store at room temperature

23. Pellet and crushed/powdered dry ice

24. $4 \%$ Paraformaldehyde (PFA) in PBS (Boston BioProducts, catalog number: BM-155), store at $4{ }^{\circ} \mathrm{C}$ for short term storage or $-20^{\circ} \mathrm{C}$ for long term storage

25. Triton X-100 (Sigma, catalog number: T-8787)

26. Rabbit anti-PDGFR beta antibody (Abcam, catalog number: ab32570)

27. FITC-BSL1: Fluorescein labeled Griffonia (Bandeiraea) Simplicifolia Lectin I (GSL I, BSL I), (Vector labraotries, catalog number: $\mathrm{F}-1101$ ), store at $4{ }^{\circ} \mathrm{C}$ protected from light

28. Antifade Mounting Medium with DAPI (Vectasheild, catalog number: $\mathrm{H}-1200$ ), store at $4{ }^{\circ} \mathrm{C}$ protected from light

29. Trizma Base (Sigma, catalog number: T1503)

30. Proteinase K (QIAGEN, catalog number: 19131), store at room temperature

31. EDTA (Sigma, catalog number: EDS)

32. Glycerol (Sigma, catalog number: G5516)

33. Fetal bovine serum (FBS) (Sigma, catalog number: F4135)

34. Trypsin digestion buffer (see Recipes) 
35. $6 \mathrm{mg} / \mathrm{ml}$ FITC-Na Solution (see Recipes)

36. $20 \mathrm{mg} / \mathrm{ml} 70 \mathrm{kDa}$ FITC-dextran Solution (see Recipes)

37. $1.5 \mathrm{mg} / \mathrm{ml}$ BSA-594 (see Recipes)

38. 1x PBS (see Recipes)

39. $30 \%$ sucrose (any brand) solution in PBS, store at $4{ }^{\circ} \mathrm{C}$ for up to four weeks (see Recipes)

40. Antibody Diluent (see Recipes)

41. Cy3 conjugated AffiniPure Goat Anti-Mouse IgG $(\mathrm{H}+\mathrm{L})$ (Jackson ImmunoResearch Laboratories, catalog number: $115-165-003$ ), store at $-20^{\circ} \mathrm{C}$ (see Recipes)

42. FITC conjugated AffiniPure Donkey Anti-Rabbit IgG $(\mathrm{H}+\mathrm{L})$ (Jackson ImmunoResearch Laboratories, catalog number: $711-095-152)$, store at $-20^{\circ} \mathrm{C}$ (see Recipes)

43. Proteinase $\mathrm{K}$ antigen retrieval solution (see Recipes)

\section{Equipment}

1. $-80^{\circ} \mathrm{C}$ freezer

2. Tabletop microcentrifuge (Beckman Coulter, model: Microfuge 18 centrifuge, catalog number: 367160)

3. Tube Shaker and Rotator (Thermo Scientific, model: 4002110Q)

4. Surgical equipment for transcardial perfusion and brain harvest (Gage et al., 2012; Sultan, 2013)

5. Analytical balance that can measure micrograms (Sartorius, catalog number: CPA2202S)

6. Tabletop vortexer (Scientific Industries, catalog number: (G560)SI-0236)

7. Centrifuge that can accommodate $15 \mathrm{ml}$ conical tubes (Eppendorf, model: $5810 \mathrm{R}$, catalog number: 022625101)

8. Spectrophotometer (Eppendorf, model: BioPhotometer Plus, catalog number: 6132)

9. Cuvets (UVette Cuvets) (Eppendorf, catalog number: 952010069)

10. Transcardial perfusion apparatus (Gage et al., 2012)

11. Styrofoam cooler or equivalent containers to hold dry ice

12. Stainless steel tray or equivalent that can fit into container holding dry ice

13. Slide warmer (Marshall Scientific, model: 26020)

14. Cryostat (Leica, model: CM3050 S)

15. Fluorescent microscope with green (FITC), red (Cy3), and blue (DAPI) channels and camera (Zeiss Axioskop2 Plus upright microscope and Spot RT3 camera with Spot RT3 camera and Spot Imaging software)

\section{Software}

1. Spot Imaging software

2. Adobe Photoshop 


\section{Procedure}

\section{A. ETX Activation}

1. Use BSL2 precautions while activating ETX.

2. Thaw one vial of epsilon protoxin $(500 \mu \mathrm{l}$ per vial) at room temperature. Concentration of epsilon protoxin is $0.5 \mathrm{mg} / \mathrm{ml}$ or $15 \mu \mathrm{M}$.

3. Prepare trypsin resin.

a. Resuspend resin by vortexing or pipetting.

b. Transfer $125 \mu \mathrm{l}$ of trypsin resin to microcentrifuge tube.

c. Wash trypsin with digestion buffer.

i. Add $500 \mu$ l of digestion buffer to tube.

ii. Centrifuge for ten $\min$ at $18,000 \times g$.

iii. Remove supernatant and discard.

d. Repeat twice for a total of three washes.

4. Transfer $500 \mu \mathrm{l}$ of thawed epsilon protoxin to washed trypsin-resin. Carefully suspend resin by pipetting up and down.

5. Incubate at $37^{\circ} \mathrm{C}$ for two hours with gentle agitation with a tube shaker and rotator.

6. After incubation, centrifuge at $18,000 \times g$ for $10 \mathrm{~min}$.

7. Transfer $400 \mu \mathrm{l}$ of supernatant to new microcentrifuge tube and discard remainder and resin pellet. The supernatant contains the activated ETX.

8. Aliquot activated ETX into 10 or $20 \mu \mathrm{l}$ aliquots and store at $-80^{\circ} \mathrm{C}$ for up to six months.

9. The concentration of the activated ETX is estimated to be $337 \mu \mathrm{g} / \mathrm{ml}$ or $11 \mu \mathrm{M}$ (based on concentration of epsilon protoxin and volume dilutions).

NOTE: We suggest confirming activation and ETX potency by testing each activated ETX vial via cell death assay on ETX-sensitive cells such as MDCK or CHO-rMAL cells (Shortt et al., 2000; Rumah et al., 2015). Complete cleavage of the $33 \mathrm{kDa}$ epsilon protoxin into the $29 \mathrm{kDa}$ active toxin can also be evaluated via western blot analysis (Linden et al., 2018).

B. ETX Induced BBB Permeability

Notes:

a. For our experiments, BBB permeability is induced by treating mice with activated ETX at a lethal dose of $5 \mathrm{ng}$ of ETX per gram body weight mouse ( $5 \mathrm{mg} / \mathrm{kg}$ ) via intraperitoneal (IP) injection. This dose normally induces moribund symptoms in 1-3 h but may be dependent on mouse strain, weight, age, and sex. When female C57BL/6 mice (8 weeks old, 18-20 g) are treated with $5 \mathrm{ng}$ of ETX per gram body weight mouse, we see mild symptoms of BBB permeability one-hour post ETX-treatment. Mild symptoms include reduced activity; specifically, mice explore their cage and surroundings less than control treated mice. Symptoms become moribund approximately $3 \mathrm{~h}$ after ETX treatment. Moribund symptoms include severe depression/lethargy and occasionally, convulsions. Mice experiencing severe depression/lethargy will not move if gently 
nudged and only display escape behavior when manually restrained. It should be noted that we have never observed mice recover from mild symptoms using this dose. All mice displaying mild symptoms eventually become moribund. Therefore, symptomatic mice, even those with mild symptoms, need to be carefully observed. We recommend that researches perform preliminary experiments with their own murine population to determine the optimal ETX-treatment time.

$b$. For the most consistent and comparable results, we recommend using mice with similar weights, preferably within a $2 \mathrm{~g}$ range. ETX dose is based on the weight of the entire animal, so treating animals with similar weights ensures consistent total ETX doses.

1. Use BSL2 precautions when working with ETX.

2. Prepare a $500 \mathrm{ng} / \mathrm{ml}$ ETX working solution in sterile saline. Thaw a frozen aliquot of activated ETX at room temperature and dilute $1: 674(337 \mu \mathrm{g} / \mathrm{ml}$ stock solution divided by $500 \mathrm{ng} / \mathrm{ml}$ working solution) in sterile saline.

3. Immediately prior to treatment, weigh each mouse.

4. For ETX treatment, inject mice with $5 \mathrm{ng}$ of ETX per gram body weight diluted in sterile saline via IP injection with a 29-gauge insulin syringe. Briefly, restrain mouse using one hand and grasp excess skin around the nape of the neck with thumb and forefinger and place the tail between the third and fourth finger. Tilt the animal head-down at a 45-degree angle. In the lower right quadrant of the abdomen, insert syringe at a 90-degree angle to the body and administer solution. For more detailed instructions on how to perform an IP injection, refer to previously published methods (Machholz et al., 2012).

For example, a $20 \mathrm{~g}$ mouse would receive a total of $100 \mathrm{ng}$ ETX via injection of $200 \mu \mathrm{l}$ of a $500 \mathrm{ng} / \mathrm{ml}$ ETX solution.

a. $20 \mathrm{~g}$ mouse multiplied by $5 \mathrm{ng}$ of ETX per gram of mouse $=100 \mathrm{ng}$ total ETX

b. $100 \mathrm{ng}$ total ETX divided by $500 \mathrm{ng} / \mathrm{ml}=200 \mu \mathrm{l}$ of $500 \mathrm{ng} / \mathrm{ml}$ of ETX solution

5. For control treatment, inject mice with an equivalent amount of sterile saline solution via IP.

6. Observe mice for moribund symptoms. Most common symptoms are severe depression/lethargy. Mice experiencing severe depression/lethargy will not move if gently nudged and only display escape behavior when being manually restrained.

C. Evaluation of BBB permeability with FITC-Na

Note: This experiment evaluates BBB permeability to substances 376Da and smaller. This technique also allows relatively easy evaluation of BBB permeability for the entire brain without transcardial perfusion or tissue sectioning. However, this technique will not identify specific neuroanatomical locations of BBB permeability. This method could be adapted to use other FITCconjugated tracers as well.

1. Preparation prior to treatment and tissue harvest 
a. For this experiment, set up analytical balance near animal dissection area with weigh boats conveniently accessible.

b. Prepare $15 \mathrm{ml}$ conical tubes containing approximately 10-15 $2 \mathrm{~mm}$-glass beads and $1 \mathrm{ml}$ PBS. Prepare one tube per animal. Store at room temperature.

c. Prepare a $6 \mathrm{mg} / \mathrm{ml}$ FITC-Na solution in sterile saline. FITC-Na solution should be prepared fresh daily and stored in a container protected from light at room temperature.

d. Create a FITC-Na standard curve in PBS with the following concentrations: $6 \mu \mathrm{g} / \mathrm{ml}, 3 \mu \mathrm{g} / \mathrm{ml}$, $1.5 \mu \mathrm{g} / \mathrm{ml}, 0.75 \mu \mathrm{g} / \mathrm{ml}, 0.375 \mu \mathrm{g} / \mathrm{ml}, 0.186 \mu \mathrm{g} / \mathrm{ml}$, and $0.094 \mu \mathrm{g} / \mathrm{ml}$. Measure and record absorbance for each standard using a spectrophotometer at $490 \mathrm{~nm}$ or equivalent. A new standard curve should be generated each day with the freshly prepared $6 \mathrm{mg} / \mathrm{ml} \mathrm{FITC-Na}$ solution.

2. Inject mice with FITC-Na

a. Treat mice with ETX as described above for the desired amount of time.

b. Using the $6 \mathrm{mg} / \mathrm{ml}$ FITC-Na solution, intravenously (IV) inject mice with $60 \mu \mathrm{g}$ of FITC-Na per gram body weight via the tail vein using a 29-gauge insulin needle. Briefly, place animal in a cylindrical restraint tube. Warm entire animal or tail to vasodilate blood vessels in the tail. Insert needle in the lateral tail vein near the distal third of the tail. Slowly inject the material. If the needle has been properly inserted into the vein, no resistance should occur as the plunger is depressed and the solution is injected. In addition, the vein should temporarily clear of blood as the solution is injected. For more detailed instructions on how to perform an IV injection, please refer to previously published methods (Gage et al., 2012). For example, a $20 \mathrm{~g}$ mouse would receive a total of $1.2 \mathrm{mg}$ of FITC-Na via injection of $200 \mu \mathrm{l}$ of a $6 \mathrm{mg} / \mathrm{ml}$ FITC-Na solution.

i. $20 \mathrm{~g}$ mouse multiplied by $60 \mu \mathrm{g}$ of FITC-Na per gram of mouse $=1.2 \mathrm{mg}$ total FITC-Na

ii. $1.2 \mathrm{mg}$ of total FITC-Na divided by $6 \mathrm{mg} / \mathrm{ml} \mathrm{FITC-Na} \mathrm{solution}=200 \mu \mathrm{l}$ of $6 \mathrm{mg} / \mathrm{ml}$ FITCNa solution

c. Observe animal for 10 min post FITC-Na administration.

d. Euthanize animals with a lethal dose of xylazine and ketamine cocktail $(450 \mathrm{mg} / \mathrm{kg}$ and $45 \mathrm{mg} / \mathrm{kg}$ ) via IP injection with 29-gauge insulin needle. Lethal doses of anesthetic agents typically occur at three times the surgical dose. In our hands, death typically occurs $30 \mathrm{~min}$ after administration. It is important to note that carbon dioxide administration, as a method of euthanasia, is not appropriate for BBB permeability studies, as carbon dioxide can cause BBB permeability (Clemedson et al., 1956 and 1958).

3. FITC-Na measurement

a. After the mouse has expired (absence of heartbeat), allow the animal to "rest" for $5 \mathrm{~min}$ prior to decapitation to prevent exsanguination.

b. Spray mouse liberally with $70 \%$ ethanol to wet down fur.

c. Decapitate mouse and remove whole brain. For detailed instructions on how to perform brain dissection, please refer to previously published methods (Sultan, 2013). 
d. Place a fresh weigh boat on analytical scale and tare or zero the instrument.

e. Transfer the whole brain to weigh boat on analytical scale and record the weight of the harvested brain.

f. Using a fresh razor blade, mince brain tissue into approximately 1-2 mm chunks and transfer to $15 \mathrm{ml}$ conical tube containing glass beads and $1 \mathrm{ml} \mathrm{PBS}$. Store at room temperature until all brains have been harvested.

g. Secure conical caps and vortex aggressively at top setting for two min.

h. Repeat for all remaining animals.

i. Centrifuge $15 \mathrm{ml}$ conical tubes containing homogenized brains at $1,500 \times \mathrm{g}$ for ten min to pellet beads.

j. Transfer supernatant to microcentrifuge tube and centrifuge at $18,000 \times g$ to clear cellular debris.

k. Transfer cleared supernatant to new microcentrifuge tube.

I. Measure absorbance of cleared supernatant at $490 \mathrm{~nm}$ or equivalent using a spectrophotometer.

m. Calculate the concentration of FITC-Na in cleared supernatants using the FITC-Na standard curve generated previously.

n. Calculate the amount of FITC-Na per gram of harvested brain by dividing the FITC-Na concentration calculated in the above step by the weight of the harvested brain $(\mu \mathrm{g} / \mathrm{ml} / \mathrm{brain})$.

o. Alternatively, results may be normalized to control-treated mice and expressed as FITC-Na Extraversion (\% CT) (Figure 2A).

For example, because our experiment was conducted over multiple days, and each day requires a fresh FITC-Na solution be prepared, each ETX-treated mouse was matched with a saline-treated control animal that was performed on the same day. FITC-Na concentrations from each ETX-treated mouse are normalized (divided by) to the FITC-Na concentration of their saline-treated control match. Saline-treated control mice are normalized (divided) to themselves. Express results as a percentage and calculate mean and standard deviation for each treatment group.

Alternatively, if animals are all processed on the same day, calculate the mean FITC-Na concentration ( $\mu \mathrm{g} / \mathrm{ml} / \mathrm{brain})$ for the control group. Normalize (divide) each value from both the control and ETX treated group by the mean control value. Express results as a percentage and calculate mean and standard deviation for each treatment group. 
A

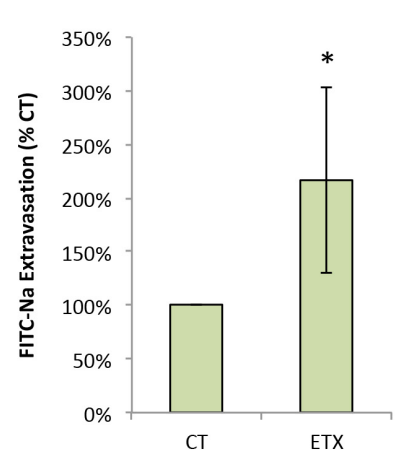

B

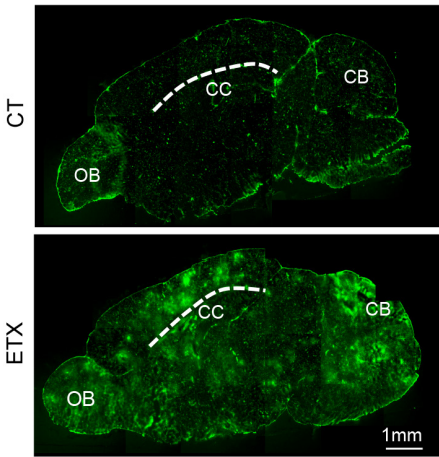

C

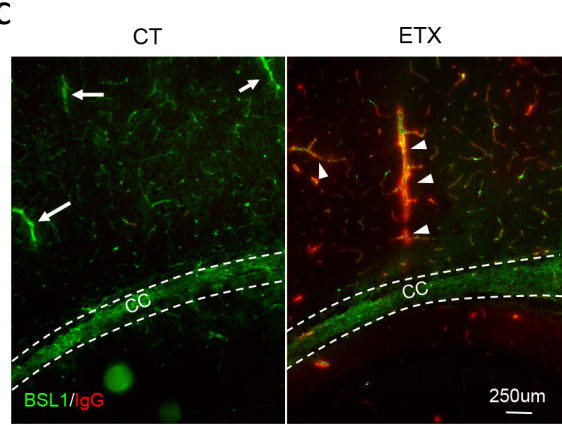

D

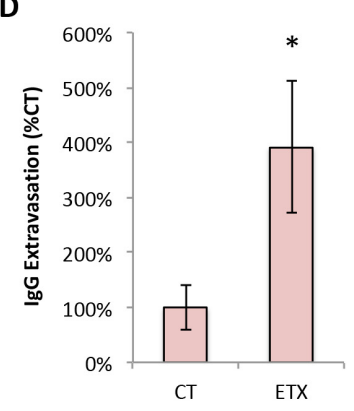

Figure 2. Representative evaluation of BBB permeability using the described methods. Results reprinted from Linden et al., 2019. Mice were treated with ETX or saline (CT) as described. A. BBB permeability assessed using FITC-Na as a tracer followed by brain homogenization and FITC-Na measurement via spectrophotometry. *Results expressed as mean \pm STDEV, $P<0.05$ determined by unpaired $t$-test. B. Micrographs of BBB permeability accessed using $70 \mathrm{kDa}$ FITC-dextran and fluorescent microscopy. Sections are approximately 1.5 millimeters to the left of the mid-sagittal plane. Dashed line identifies corpus callosum (CC). OB, olfactory bulb. CB, Cerebellum. C. Micrographs of BBB permeability accessed by endogenous IgG (red) staining. Sagittal sections of PBS only perfused animals. FITC-BSL1 (green) is used to identify microvasculature. Dashed lines identify width of corpus callosum (CC). White arrows indicate large vessels with no observable IgG extravasation. White arrowheads indicate areas of IgG extravasations around large vessels. D. Semi-quantitative measurements of IgG extravasation in control and ETX treated mice, measured by fluorescent intensity. *Results expressed as mean \pm STDEV, $P=0.05$ determined by unpaired $t$-test.

D. Evaluation of BBB permeability using $70 \mathrm{kDa}$ FITC-dextran

Note: Use of exogenous $70 \mathrm{kDa}$ FITC-dextran as a tracer for BBB permeability studies evaluates extravasation of materials $70 \mathrm{kDa}$ or less. FITC-dextran with different molecular weights $(4,000$, 40,000, and 150,000 kDa) may also be used for this procedure. FITC-Dextran is not fixable by PFA, therefore cardiac perfusion is not recommended with this tracer. In addition, we recommend tissue sections be imaged "dry", without the use of fixatives, staining procedures, or mounting media, as use of any aqueous material or solvents may cause leaching of this tracer (Hoffmann et al., 2011). 
Although this is only a qualitative method for measuring BBB permeability, it works well to identify specific neuroanatomical locations of BBB permeability. In control tissue, $70 \mathrm{kDa}$ FITC-dextran should be confined to vascular lumen, with some extravasation in neuroanatomical areas of known BBB permeability. In brains with dysfunctional BBB activity, large accumulations or "halos" of $70 \mathrm{kDa}$ FITC-dextran occur in the brain parenchyma and are considered signs of BBB permeability.

1. Preparation prior to treatment and tissue harvest:

a. Prepare a $20 \mathrm{mg} / \mathrm{ml} 70 \mathrm{kDa}$ FITC-Dextran in sterile saline. Store at $4{ }^{\circ} \mathrm{C}$ for up to one month and protect from light. Pre-warm solution to room temperature or $37{ }^{\circ} \mathrm{C}$ before administration to animals.

b. Prepare materials for freezing harvested brains by pre-chilling metal plate on dry ice pellets in a container for at least 20 min before use.

c. If necessary, crush dry ice pellets to create dry ice powder and store in container until use.

2. Inject mice with $70 \mathrm{kDa}$ FITC-dextran

a. Treat mice with ETX as described above for the desired amount of time.

b. Administer $0.2 \mathrm{mg}$ of $70 \mathrm{kDa}$ FITC-dextran per gram body weight via the tail vein IV injection using a 29-gauge insulin needle and the $20 \mathrm{mg} / \mathrm{ml} 70 \mathrm{kDa}$ FITC-dextran solution. Briefly, place animal in a cylindrical restraint tube. Warm entire animal or tail to vasodilate blood vessels in the tail. Insert needle in the lateral tail vein near the distal third of the tail. Slowly inject the material. If the needle has been properly inserted into the vein, no resistance should occur as the plunger is depressed and the solution is injected. In addition, the vein should temporarily clear of blood as the solution is injected. For more detailed instructions on how to perform an IV injection, refer to previously published methods (Machholz et al., 2012).

For example, a $20 \mathrm{~g}$ mouse would receive a total of $4 \mathrm{mg}$ of $70 \mathrm{kDa}$ FITC-dextran via injection of $200 \mu \mathrm{l}$ of a $20 \mathrm{mg} / \mathrm{ml} 70 \mathrm{kDa}$ FITC-dextran solution

i. $20 \mathrm{~g}$ mouse multiplied by $0.2 \mathrm{mg} / \mathrm{ml}$ of $70 \mathrm{kDa}$ FITC-dextran per gram of mouse $=4 \mathrm{mg}$ total $70 \mathrm{kDa}$ FITC-dextran

ii. $4 \mathrm{mg}$ total $70 \mathrm{kDa}$ FITC-dextran divided by $20 \mathrm{mg} / \mathrm{ml} 70 \mathrm{kDa}$ FITC-dextran solution = $200 \mu \mathrm{l}$ of $20 \mathrm{mg} / \mathrm{ml} 70 \mathrm{kDa}$ FITC-dextran solution

c. Observe animal for 10 min post $70 \mathrm{kDa}$ FITC-dextran administration.

d. Euthanize animals with a lethal dose of xylazine and ketamine cocktail $(450 \mathrm{mg} / \mathrm{kg}$ and $45 \mathrm{mg} / \mathrm{kg}$ ) via IP injection with 29-gauge insulin pen. Lethal doses of anesthetic agents typically occur at three times the surgical dose. In our hands, death typically occurs $30 \mathrm{~min}$ after administration. It is important to note that carbon dioxide administration, as a method of euthanasia, is not appropriate for BBB permeability studies, as carbon dioxide administration can cause BBB permeability (Clemedson et al., 1956 and 1958).

3. Brain harvest and freezing 
a. After mouse has expired (absence of heart-beat), allow the animal to "rest" for 5 min prior to decapitation to prevent exsanguination.

b. Spray mouse liberally with $70 \%$ ethanol to wet down fur.

c. Decapitate mouse and remove whole brain. For detailed instructions on how to perform brain dissection, please refer to previously published methods (Sultan, 2013).

d. Line the bottom of a labeled cryomold with a thin layer of OCT medium.

e. Gently transfer brain to OCT-lined cryomold, ventral side down.

f. While avoiding bubbles, fill cryomold with OCT until the brain tissue is completely covered.

g. Transfer cryomold to pre-chilled metal plate on dry ice in container. Quickly surround all four lateral sides of the cryomold with powered/crushed dry-ice.

h. Once the OCT has turned opaque, the frozen specimen may be stored on dry-ice pellets while other brains are harvested and frozen.

i. Wrap the frozen specimen within the cryomold in aluminum foil and transfer to $-20^{\circ} \mathrm{C}$ for storage.

4. Tissue sectioning and Imaging

a. Number microscope slides with pencil, one through 150.

b. Section tissue in $16 \mu \mathrm{m}$ slices along the sagittal plane moving from the left lateral side toward the mid-line, transferring four serial sections onto one microscope slide, starting with slide one (Figure 3).

A

$$
\text { Rostral }
$$

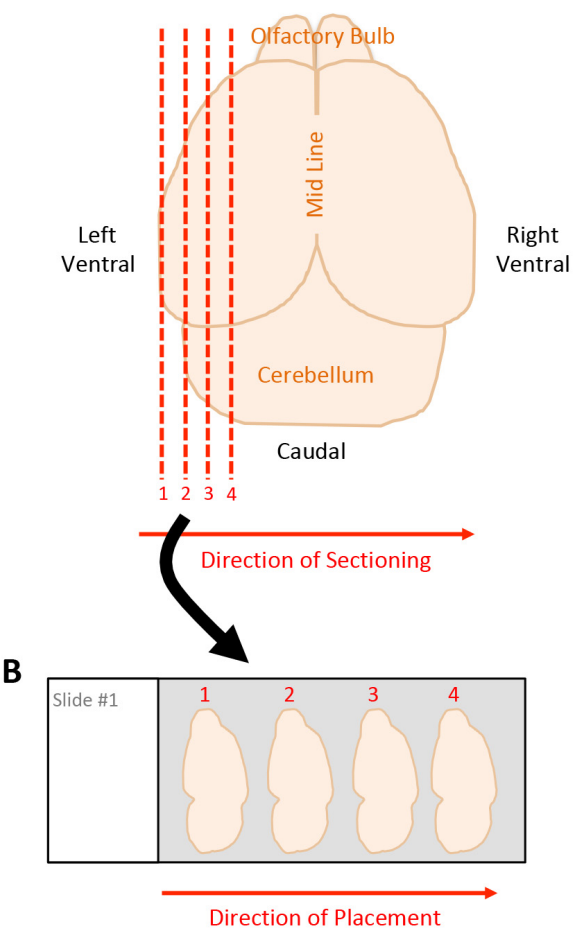

Figure 3. Schematic of tissue sectioning strategy. A. Dorsal view of harvested mouse brain. Red dashed lines indicate sagittal sections beginning on the left ventral side moving 
towards the midline, starting with section 1 through 4. B. Placement of tissue sections onto microscope slide, moving left to right, starting with section 1 through 4.

c. Place slides into storage box and continue sectioning until desired amount of brain has been sampled. It typically takes 150 slides to section an entire brain using this method.

d. Sectioned samples may be stored at $-20^{\circ} \mathrm{C}$ until imaging. However, we suggest imaging immediately after sectioning, as chilled slides may gather condensation as they thaw, causing the fluorescent tracer to bleed.

e. Examine slides on fluorescent microscope using the green (FITC) channel.

f. To make accurate comparisons, it's desirable to compare similar neuroanatomical locations between different animals and different treatment groups. Use the numbered slides to locate similar anatomical areas for comparisons. As with all microscopic comparisons, ensure that the same microscope (magnification) and imaging settings (exposure, etc.) are used for all comparisons (Figure 2B).

g. For our experiments, tissues are imaged using Zeiss Axioskop2 Plus upright microscope and Spot RT3 camera and software and post-processed using identical settings in Adobe Photoshop. Typically, images are captured at $100 x$ or $200 x$ magnification in gray scale monochrome using the fluorescence image type with default settings and manual exposure. Exposure times should be identified independently for each fluorophore and experiment. To select the best exposure times, we suggest utilizing the feature that allows you to indicate saturated pixels in monochrome. This feature can help reduce data loss by preventing too many saturated pixels. When enabled, this feature indicates saturated pixels in red. By using this feature, one can select an exposure time that minimizes the amount of red (saturated) pixels while allowing negative areas to be black.

\section{E. Evaluation of BBB Permeability Using Endogenous IgG}

Note: In this method, endogenous IgG is used as a marker of BBB permeability. This evaluates extravasation of substances that are $155 \mathrm{kDa}$ or smaller. Animals are perfused with PBS only to remove luminal IgG. Because this method involves freezing of fresh, PBS perfused only tissue, sectioned tissue can be stained using a wide array of immunofluorescent assays (IFA) and IHC applications without the use of antigen retrieval. However, the morphology of sectioned tissue is inferior to tissue that has been perfused with fixative. Because animals are perfused, all luminal lgG will be removed. Therefore, any subsequent IgG signal observed in brain parenchyma or colocalizing with vasculature should be considered a sign of BBB permeability. IgG observed colocalizing with vasculature may indicate BEC internalization of IgG or IgG deposition on vascular walls.

1. Preparation prior to treatment and tissue harvest

a. Prepare pump apparatus and PBS reagent for transcardial perfusion (Gage et al., 2012). 
b. Prepare materials for freezing harvested brains by pre-chilling metal plate on dry ice pellets in container for at least 20 min before use.

c. If necessary, crush dry ice pellets to create dry ice powder and store in container until use.

2. Transcardial perfusion and brain harvest

a. Treat mice with ETX as described above for the desired amount of time.

b. Deeply anesthetize animals with a surgical dose of xylazine and ketamine cocktail (150 mg/kg and $15 \mathrm{mg} / \mathrm{kg}$ ) via IP injection with 29-gauge insulin needle. Full anesthetization typically happens within 20 min.

c. Perform trasncardial perfusion with at least $20 \mathrm{ml}$ of PBS as previously published (Gage et al., 2012). Briefly, place the mouse in a supine position with legs extended. Make a midline incision into the abdomen to the ribcage followed by an intersecting lateral incision below the ribcage, exposing the abdominal cavity. Be careful not to nick any internal organs, especially the liver. Gently separate the liver from the diaphragm by gently nudging the liver away from the diaphragm. Carefully cut the diaphragm along its length, exposing the pleural cavity. Cut through the ribcage on both the right and left sides, flipping the sternum up and away, towards the head, carefully trimming any tissue connected to heart. Insert a needle connected to the pump apparatus and PBS into the posterior end of the left ventricle up into the ascending aorta. Secure needle with hemostat and make a small incision on the right atrium. Pump at least $20 \mathrm{ml}$ of PBS through the animal. Blood should be observed exiting the right atrium, gradually clearing to PBS. A sign that the mouse has been adequately perfused is the desaturation of the liver from a dark red to light brown color.

d. After perfusion, spray mouse liberally with $70 \%$ ethanol to wet down fur.

e. Decapitate mouse and remove whole brain. For detailed instructions on how to perform brain dissection, please refer to previously published methods (Sultan, 2013).

f. Line the bottom of a labeled cryomold with a thin layer of OCT.

g. Gently transfer brain to OCT-lined cryomold, ventral side down.

h. While avoiding bubbles, fill cryomold with OCT until the brain tissue is completely covered.

i. Transfer cryomold to pre-chilled metal plate on dry ice in container. Quickly surround all four lateral sides of the cryomold with powered/crushed dry-ice.

j. Once the OCT has turned opaque, the frozen specimen may be stored on dry-ice pellets while other mice are perfused, etc.

k. Wrap the frozen specimen within the cryomold in aluminum foil and transfer to $-20^{\circ} \mathrm{C}$ for storage.

3. Tissue sectioning

a. Number microscope slides one through 150 using a pencil. Do not use ink-based writing pens, as various fixatives will cause the ink to bleed and possible result in subsequent staining artifacts.

b. Section tissue in $16 \mu \mathrm{m}$ sections along the sagittal plane moving from the left lateral side toward the mid-line, transferring four serial sections onto one microscope slide, starting with 
slide one (Figure 3).

c. Place slides into storage box and continue sectioning until desired amount of brain has been sampled. It typically takes 150 slides to section an entire brain using this method.

d. Slides may be stored at $-20^{\circ} \mathrm{C}$.

4. Tissue staining with FITC-BSL1 and anti-mouse IgG

a. Remove slides from $-20^{\circ} \mathrm{C}$ and allow to warm to room temperature.

b. To increase tissue adherence during staining, pre-heat slides at $55^{\circ} \mathrm{C}$ on a slide warmer for 20 min before staining. Allow to cool to room temperature.

c. Fix slides in $4 \%$ PFA/PBS for ten min at room temperature.

d. Wash slides three times in PBS for five min at room temperature.

e. Block in antibody diluent for $30 \mathrm{~min}$ at room temperature.

f. While sections block, prepare staining solution by diluting Сy3 anti-mouse IgG antibody 1:500 and FITC-BSL1 1:200 in the same antibody diluent.

g. Wash slides twice in PBS for five min at room temperature.

h. Incubate slides with prepared staining solution for one hour at room temperature or overnight at $4{ }^{\circ} \mathrm{C}$.

i. Wash slides three times in PBS for five min at room temperature.

j. Apply mounting media with DAPI to slides and coverslip.

k. Using Kimwipes or equivalent, gently blot excess mounting media with DAPI off of slides.

I. Seal edges of coverslips with clear nail polish.

$\mathrm{m}$. Stained slides may be stored at $4{ }^{\circ} \mathrm{C}$ until imaging. Allow slides to warm to room temperature before imaging.

h. Examine slides on fluorescent microscope using the green (FITC) to visualize vasculature, the red (Cy3) channel to visualize IgG, and the blue (DAPI) channel to visualize nuclear staining (Figure $2 \mathrm{C}$ ). For our experiments, tissues are imaged using Zeiss Axioskop2 Plus upright microscope and Spot RT3 camera and software and post-processed using identical settings in Adobe Photoshop. Typically, images are captured at 200x or 400x magnification in gray scale monochrome using the fluorescence image type with default settings and manual exposure. Exposure times should be identified independently for each fluorophore and experiment. To select the best exposure times, we suggest utilizing the feature that allows you to indicate saturated pixels in monochrome. This feature can help reduce data loss by preventing too many saturated pixels. When enabled, this feature indicates saturated pixels in red. By using this feature, one can select an exposure time, which minimizes the amount of red (saturated) pixels while allowing negative areas to be black.

5. Measurement of IgG extravasation

a. To compare IgG permeability between different treatment groups, identical neuroanatomical locations from different mice must be examined. Use the numbered slides and anatomical landmarks delineated by nuclear DAPI staining and vascular FITC-BSL1 staining to locate desired areas. For each treatment condition, we recommend that a minimum of three tissue 
sections from each animal and three animals per condition be examined. In addition, all control and ETX-treated slides need to be stained simultaneously using the same prepared reagents. As with all microscopic comparisons, ensure that the same microscope (magnification) and imaging settings (exposure, etc.) are used for all comparisons.

For example, in our previously published experiment, we were interested in $\lg$ permeability in pericallosal tissue (Linden et al., 2019). Images were captured in the myelinated region of the cerebral cortex, superior to the hippocampus and lateral ventricle for all mice, spanning from the corpus callosum to subcortical gray matter.

To measure IgG permeability, capture images on the red (Су3) channel using a fluorescent microscope. For our experiments, tissues are imaged using Zeiss Axioskop2 Plus upright microscope and Spot RT3 camera and software. Typically, images are captured at 200x magnification in gray scale monochrome using the fluorescence image type with default settings and manual exposure. Exposure times should be identified independently for each fluorophore and experiment. To select the best exposure times, we suggest utilizing the feature that allows you to indicate saturated pixels in monochrome. This feature can help reduce data loss by preventing too many saturated pixels. When enabled, this feature indicates saturated pixels in red. By using this feature, one can select an exposure time, which minimizes the amount of red (saturated) pixels while allowing negative areas to be black.

b. To measure relative IgG fluorescence between animals and different conditions, import images into Image 64 and convert into an 8-bit gray format.

c. Adjust threshold identically for all imported images.

d. Calculate the mean and integrated density for each individual image for use as a measurement of fluorescence density.

e. Calculate the mean fluorescent density for each individual mouse.

f. Calculate the mean fluorescent density for entire treatment group.

g. Alternatively, normalize the results to control treatment and express results as IgG Extravasation (\% CT) (Figure 2D).

For example, calculate the mean fluorescent density for the control group. Normalize (divide) each value from both the control and ETX treated group by the mean control value. Express results as a percentage and calculate mean and standard deviation for each treatment group.

\section{Optional: Immunofluorescent staining procedure}

NOTE: In this procedure, we will describe how to stain for both endogenous IgG leakage and pericytes using a rabbit anti-PDGFbeta marker. Use of a pericyte marker can help identify areas of IgG permeability that extend beyond endothelial cells. Alternate antibodies may be substituted for this procedure. When selecting antibodies to use, ensure that the primary antibody is not of mouse origin; we have the most success using rabbit antibodies. We also 
highly suggest that the secondary antibodies used for this procedure (in our case, FITC conjugated donkey anti-rabbit $\lg G$ ) be pre-absorbed to mouse $\lg G$ to minimize cross-reaction.

a. Remove slides from $-20^{\circ} \mathrm{C}$ and allow to warm to room temperature.

b. To increase tissue adherence during staining, pre-heat slides at $55^{\circ} \mathrm{C}$ on a slide warmer for 20 min before staining. Allow to cool to room temperature.

c. Fix slides in $4 \%$ PFA/PBS for ten min at room temperature.

d. Wash slides three times in PBS for five min at room temperature.

e. Block in antibody diluent for $30 \mathrm{~min}$ at room temperature.

f. While sections block, prepare primary staining solution by diluting rabbit anti-PDGFR beta antibody 1:100 in antibody diluent.

g. Wash slides twice in PBS for five min at room temperature.

h. Incubate slides with prepared primary staining solution for one hour at room temperature or overnight at $4{ }^{\circ} \mathrm{C}$.

i. Wash slides three times in PBS for five min at room temperature.

j. While slides wash, prepare the secondary staining solution by diluting the Cy3 anti-mouse IgG antibody 1:500 and the FITC anti-rabbit IgG antibody 1:500 in the same antibody diluent.

k. Incubate slides with prepared secondary staining solution for one hour at room temperature or overnight at $4{ }^{\circ} \mathrm{C}$.

I. Wash slides three times in PBS for five min at room temperature.

m. Apply mounting media with DAPI to slides and coverslip.

n. Using Kimwipes or equivalent, gently blot excess mounting media with DAPI off of slides.

o. Seal edges of coverslips with clear nail polish.

p. Stained slides may be stored at $4{ }^{\circ} \mathrm{C}$ until imaging. Allow slides to warm to room temperature before imaging.

i. Examine slides on fluorescent microscope using the green (FITC) to visualize pericytes, the red (Су3) channel to visualize IgG, and the blue DAPI channel to visualize nuclear staining. For our experiments, tissues are imaged using Zeiss Axioskop2 Plus upright microscope and Spot RT3 camera and software and post-processed using identical settings in Adobe Photoshop. Typically, images are captured at $200 x$ or $400 x$ magnification in gray scale monochrome using the fluorescence image type with default settings and manual exposure. Exposure times should be identified independently for each fluorophore and experiment. To select the best exposure times, we suggest utilizing the feature that allows you to indicate saturated pixels in monochrome. This feature can help reduce data loss by preventing too many saturated pixels. When enabled, this feature indicates saturated pixels in red. By using this feature, one can select an exposure time, which minimizes the amount of red (saturated) pixels while allowing negative areas to be black.

\section{F. Evaluation of BBB permeability using BSA-594}

Note: Use of exogenous BSA as a tracer for BBB permeability evaluates extravasation of materials 
$66.5 \mathrm{kDa}$ or less. BSA is fixable with $4 \%$ PFA, making it an ideal tracer to be used for permeability studies requiring tissue perfusion with $4 \%$ PFA/PBS. This technique, in combination with sucrose cryoprotection, results in superior morphology preservation. However, sectioned tissue prepared using this method may require antigen retrieval prior to IFA or IHC applications. Because animals are perfused, all luminal BSA-594 will be removed. Therefore, any subsequent BSA-594 signal observed in brain parenchyma or colocalizing with vasculature should be considered a sign of $B B B$ permeability. Colocalization of BSA-594 with vasculature may indicate BSA-594 internalization by BEC. Permeability to albumin is believed to be a result of increased transcytosis via caveolaedependent mechanisms (Schnitzer et al., 1994; Schubert et al., 2001; Frank et al., 2003). However, specific mechanisms of BBB permeability should be confirmed using additional experimental methods.

1. Preparation prior to treatment and tissue harvest:

a. Prepare a $1.5 \mathrm{mg} / \mathrm{ml} \mathrm{BSA}-594$ in sterile saline. Store at $4{ }^{\circ} \mathrm{C}$ for up to one month and protect from light. Pre-warm solution to room temperature or $37^{\circ} \mathrm{C}$ before administration to animals.

b. Prepare pump apparatus, PBS and 4\% PFA/PBS reagents for transcardial perfusion.

c. For each brain to be harvested, prepare $50 \mathrm{ml}$ conical tube with $10 \mathrm{ml} 4 \%$ PFA/PBS solution and a separate $50 \mathrm{ml}$ conical tube with $10 \mathrm{ml} 30 \%$ sucrose solution.

2. Inject mice with BSA-594

a. Treat mice with ETX as described above for the desired amount of time.

b. Using the $1.5 \mathrm{mg} / \mathrm{ml}$ BSA-594 solution, intravenously inject mice with $15 \mu \mathrm{g}$ of BSA-594 per gram body weight via the tail vein using a 29-gauge insulin needle. Briefly, place animal in a cylindrical restraint tube. Warm entire animal or tail to vasodilate blood vessels in the tail. Insert needle in the lateral tail vein near the distal third of the tail. Slowly inject the material. If the needle has been properly inserted into the vein, no resistance should occur as the plunger is depressed and the solution is injected. In addition, the vein should temporarily clear of blood as the solution is injected. For more detailed instructions on how to perform an IV injection, refer to previously published methods (Machholz et al., 2012).

For example, a $20 \mathrm{~g}$ mouse would receive a total of $300 \mu \mathrm{g}$ of BSA-594 via injection of $200 \mu \mathrm{l}$ of a $1.5 \mathrm{mg} / \mathrm{ml} \mathrm{BSA}-594$ solution

i. $20 \mathrm{~g}$ mouse multiplied by $15 \mu \mathrm{g}$ BSA-594 per gram of mouse $=300 \mu \mathrm{g}$ total BSA-594

ii. $300 \mu \mathrm{g}$ BSA-594 divided by $1.5 \mathrm{mg} / \mathrm{ml}$ BSA-594 solution $=200 \mu \mathrm{l}$ of $1.5 \mathrm{mg} / \mathrm{ml} \mathrm{BSA-594}$ solution

c. Observe animal for $10 \mathrm{~min}$ post treatment.

3. Transcardial perfusion and brain harvest

a. Deeply anesthetize animals with a lethal dose of xylazine and ketamine cocktail $(150 \mathrm{mg} / \mathrm{kg}$ and $15 \mathrm{mg} / \mathrm{kg}$ ) via IP injection with 29-gauge insulin needle. Full anesthetization typically happens within 20 min.

b. Perform traanscardial perfusion with at least $20 \mathrm{ml}$ of PBS followed by at least $20 \mathrm{ml}$ of $4 \%$ 
PFA/PBS solution as previously published (Gage et al., 2012). Briefly, place the mouse in a supine position with legs extended. Make a midline incision into the abdomen to the ribcage followed by an intersecting lateral incision below the ribcage, exposing the abdominal cavity. Be careful not to nick any internal organs, especially the liver. Gently separate the liver from the diaphragm by gently nudging the liver away from the diaphragm. Carefully cut the diaphragm along its length, exposing the pleural cavity. Cut through the ribcage on both the right and left sides, flipping the sternum up and away, towards the head, carefully trimming any tissue connected to heart. Insert a needle connected to the pump apparatus and PBS into the posterior end of the left ventricle up into the ascending aorta. Secure needle with hemostat and make a small incision on the right atrium. Pump at least $20 \mathrm{ml}$ of PBS through the animal. Blood should be observed exiting the right atrium, gradually clearing to PBS. A sign that the mouse has been adequately perfused is the desaturation of the liver from a dark red to light brown color. Follow with $20 \mathrm{ml}$ of $4 \%$ PFA/PBS. Fixation tremors should be observed and animal will be stiff by the end of PFA/PBS perfusion.

c. After perfusion, spray mouse liberally with $70 \%$ ethanol to wet down fur. Decapitate mouse and remove whole brain. For detailed instructions on how to perform brain dissection, refer to previously published methods (Sultan, 2013).

d. After harvest, post-fix harvested brains by transferring tissue to a $50 \mathrm{ml}$ conical tube containing $10 \mathrm{mls} 4 \% / \mathrm{PFA} / \mathrm{PBS}$ solution. Incubate $16 \mathrm{~h}$ at $4{ }^{\circ} \mathrm{C}$.

e. Transfer fixed brain tissue to $50 \mathrm{ml}$ conical tube containing $10 \mathrm{ml}$ of $30 \%$ sucrose/PBS solution. Incubate at $4{ }^{\circ} \mathrm{C}$ until tissue sinks or overnight.

4. Freezing cryoprotected brains

a. Prepare materials for freezing harvested brains by pre-chilling metal plate on dry ice pellets in container for at least $20 \mathrm{~min}$ before use.

b. If necessary, crush dry ice pellets to create dry-ice powder and store in container until use.

c. Line the bottom of a labeled cryomold with a thin layer of OCT medium.

d. Gently transfer cryoprotected brain to OCT medium-lined cryomold, ventral side down.

e. While avoiding bubbles, fill cryomold with OCT medium until the brain tissue is completely covered.

f. Transfer cryomold to pre-chilled metal plate on dry ice in container. Quickly surround all four lateral sides of the cryomold with powered/crushed dry-ice.

g. Once the OCT has turned opaque, the frozen specimen may be stored on dry-ice pellets while other brains are harvested and frozen.

h. Wrap the frozen specimen within the cryomold in aluminum foil and transfer to $-20^{\circ} \mathrm{C}$ for storage.

5. Tissue sectioning

a. Number microscope slides one through 150 using a pencil. Do not use pen, as various fixatives will cause ink to bleed and may create staining artifacts.

b. Section tissue in $16 \mu \mathrm{m}$ sections along the sagittal plane moving from the left lateral side 
toward the mid-line, transferring four serial sections onto one microscope slide, starting with slide one (Figure 3).

c. Place slides into storage box and continue sectioning until desired amount of brain has been sampled. It typically takes 150 slides to section an entire brain using this method.

d. Slides may be stored at $-20^{\circ} \mathrm{C}$ until use.

6. Tissue staining with FITC-BSL1

a. Prior to staining, prepare proteinase $\mathrm{K}$ antigen retrieval solution at a final concentration of $20 \mu \mathrm{g} / \mathrm{ml}$ in TE buffer by diluting the stock proteinase $\mathrm{K}$ solution $(20 \mathrm{mg} / \mathrm{ml}) 1: 1,000$ in TE buffer. This solution may be stored for 6 months at $4{ }^{\circ} \mathrm{C}$.

b. Remove slides from $-20^{\circ} \mathrm{C}$ and allow to warm to room temperature.

c. To increase tissue adherence to slides, pre-heat slides at $55^{\circ} \mathrm{C}$ on a slide warmer for 20 min. Allow to cool to room temperature.

d. Incubate slides in proteinase $\mathrm{K}$ antigen retrieval solution for $15 \mathrm{~min}$ at $37^{\circ} \mathrm{C}$.

e. Wash slides three times in PBS for five min at room temperature.

f. Block in antibody diluent for $30 \mathrm{~min}$ at room temperature.

g. While sections block, prepare staining solution by diluting FITC-BSL1 1:200 in antibody diluent.

h. Wash slides twice in PBS for five min at room temperature.

i. Incubate slides with prepared staining solution for one hour at room temperature or overnight at $4{ }^{\circ} \mathrm{C}$.

j. Wash slides three times in PBS for five min at room temperature.

k. Apply mounting media with DAPI to slides and coverslip.

I. Using Kimwipes, gently blot excess mounting media with DAPI off of slides.

$\mathrm{m}$. Seal edges of coverslips with clear nail polish.

n. Stained slides may be stored at $4{ }^{\circ} \mathrm{C}$ until imaging. Allow slides to warm to room temperature before imaging.

j. Examine slides on fluorescent microscope using the FITC (BSL1/vasculature), Сy3 (BSA594), and DAPI (Nuclear) channel. For our experiments, tissues are imaged using Zeiss Axioskop2 Plus upright microscope and Spot RT3 camera and software and post-processed using identical settings in Adobe Photoshop. Typically, images are captured at 200x or 400x magnification in gray scale monochrome using the fluorescence image type with default settings and manual exposure. Exposure times are identified independently for each fluorophore and experiment. To select the best exposure times, utilize the feature that allows indication of saturated pixels in monochrome. This feature can help reduce data loss by preventing too many saturated pixels. When enabled, this feature indicates saturated pixels in red. By using this feature, select an exposure time that minimizes the amount of red (saturated) pixels while allowing negative areas to be black. 


\section{Recipes}

1. Trypsin digestion buffer

$15.61 \mathrm{mg}$ Sodium phosphate monobasic dehydrate

$10 \mathrm{ml}$ deionized water

Make fresh each time

2. $6 \mathrm{mg} / \mathrm{ml} \mathrm{FITC-Na} \mathrm{Solution}$

$60 \mathrm{mg}$ Fluorescein sodium salt

$10 \mathrm{ml}$ sterile saline solution ( $0.9 \%$ sodium chloride)

Make fresh each time

3. $20 \mathrm{mg} / \mathrm{ml} 70 \mathrm{kDa}$ FITC-dextran Solution

Prepare directly in $100 \mathrm{mg}$ bottle

$100 \mathrm{mg}$ Fluorescein isothiocyanate-dextran with average molecular weight 70,000

$5 \mathrm{ml}$ sterile saline solution ( $0.9 \%$ sodium chloride)

Store at $4{ }^{\circ} \mathrm{C}$ protected from light

4. $1.5 \mathrm{mg} / \mathrm{ml}$ BSA-594

Prepare directly in $5 \mathrm{mg}$ bottle

$5 \mathrm{mg}$ Albumin from Bovine Serum, Alexa Fluor 594 conjugate

$3.333 \mathrm{ml}$ sterile saline solution ( $0.9 \%$ sodium chloride)

Store at $4{ }^{\circ} \mathrm{C}$ protected from light

5. $1 \times$ PBS

$100 \mathrm{ml} 10 x$ PBS

$900 \mathrm{ml}$ deionized water

Sterile filter

Store at room temperature

6. $30 \%$ sucrose solution

$30 \mathrm{~g}$ sucrose (and brand)

$100 \mathrm{ml}$ of $1 \times$ PBS

Store at $4{ }^{\circ} \mathrm{C}$ for up to four weeks

7. Antibody Diluent

$10 \mathrm{ml} \mathrm{FBS}$ (any brand)

$50 \mu \mathrm{l}$ triton $\mathrm{X}-100$

$40 \mathrm{ml}$ 1x PBS

Store at $4{ }^{\circ} \mathrm{C}$ for up to two weeks

8. Cy3 AffiniPure Goat Anti-Mouse IgG $(\mathrm{H}+\mathrm{L})$

Prepare $1.5 \mathrm{mg} / \mathrm{ml}$ solution directly in $2 \mathrm{mg}$ bottle

2 mg Cy3 AffiniPure Goat Anti-Mouse IgG $(\mathrm{H}+\mathrm{L})$

$750 \mu \mathrm{l}$ glycerol (any brand)

$750 \mu$ deionized water 
Store at $-20^{\circ} \mathrm{C}$ protected from light

9. FITC conjugated AffiniPure Donkey Anti-Rabbit IgG $(\mathrm{H}+\mathrm{L})$

Prepare $1.5 \mathrm{mg} / \mathrm{ml}$ solution directly in $0.5 \mathrm{mg}$ bottle

0.5 mg FITC conjugated AffiniPure Donkey Anti-Rabbit IgG $(\mathrm{H}+\mathrm{L})$

$166.5 \mu \mathrm{l}$ glycerol (any brand)

$166.5 \mu$ deionized water

Store at $-20^{\circ} \mathrm{C}$ protected from light

10. Proteinase $\mathrm{K}$ antigen retrieval solution

\section{$6.1 \mathrm{~g}$ Trizma Base}

$0.37 \mathrm{~g}$ EDTA (any brand)

$5 \mathrm{ml}$ Triton X-100

$1,000 \mathrm{ml}$ deionized water

Adjust $\mathrm{pH}$ to 8.0 using $\mathrm{HCl}$

Store at room temperature

\section{Acknowledgments}

This work, in part, was funded by the Wilma S. and Laurence A. Tisch Foundation. We thank previous work that helped influence these methods including Hoffmann et al., 2011 and Saunders et al., 2015.

\section{Competing interests}

There are no conflicts of interest or competing interest.

\section{Ethics}

All animal studies were approved by the Institutional Animal Care and Use Committee of Weill Cornell Medical College, protocol number 2012-0030. These policies and procedures have been developed based on federal regulations and laws mandated and enforced by the Animal Welfare Act, United States Department of Agriculture Animal and Plant Health Inspection Service, The National Institute of Health's Office of Laboratory Animal Welfare, the Policy of the Public Health Service, American Veterinary Medical Association Guidelines on Euthanasia, The National Institute of Health Revitalization Act, and the United States Government. Ketamine and xylazine were used for anesthesia and euthanasia for terminal in vivo studies. 


\section{$\underline{\text { References }}$}

1. Abbott, N. J. and Friedman, A. (2012). Overview and introduction: the blood-brain barrier in health and disease. Epilepsia 53 Suppl 6: 1-6.

2. Abbott, N. J., Ronnback, L. and Hansson, E. (2006). Astrocyte-endothelial interactions at the blood-brain barrier. Nat Rev Neurosci 7(1): 41-53.

3. Alvarez, J. I., Katayama, T. and Prat, A. (2013). Glial influence on the blood brain barrier. Glia 61(12): 1939-1958.

4. Balabanov, R. and Dore-Duffy, P. (1998). Role of the CNS microvascular pericyte in the bloodbrain barrier. J Neurosci Res 53(6): 637-644.

5. Buxton, D. (1978). The use of an immunoperoxidase technique to investigate by light and electron microscopy the sites of binding of Clostridium welchii type-D epsilon toxin in mice. $J$ Med Microbiol 11(3): 289-292.

6. Clemedson, C. J., Hartelius, H. and Holmberg, G. (1956). Effect of carbon dioxide inhalation on the blood-brain barrier. Nature 178(4539): 915.

7. Clemedson, C. J., Hartelius, H. and Holmberg, G. (1958). The influence of carbon dioxide inhalation on the cerebral vascular permeability to trypan blue (the blood-brain barrier). Acta Pathol Microbiol Scand 42(2): 137-149.

8. De Bock, M., Van Haver, V., Vandenbroucke, R. E., Decrock, E., Wang, N. and Leybaert, L. (2016). Into rather unexplored terrain-transcellular transport across the blood-brain barrier. Glia 64(7): 1097-1123.

9. Engelhardt, B. (2003). Development of the blood-brain barrier. Cell Tissue Res 314(1): 119-129.

10. Frank, P. G., Woodman, S. E., Park, D. S. and Lisanti, M. P. (2003). Caveolin, caveolae, and endothelial cell function. Arterioscler Thromb Vasc Biol 23(7): 1161-1168.

11. Gage, G. J., Kipke, D. R. and Shain, W. (2012). Whole animal perfusion fixation for rodents. $J$ Vis $\operatorname{Exp}(65): 3564$.

12. Gloor, S. M., Wachtel, M., Bolliger, M. F., Ishihara, H., Landmann, R. and Frei, K. (2001). Molecular and cellular permeability control at the blood-brain barrier. Brain Res Brain Res Rev 36(2-3): 258-264.

13. Hoffmann, A., Bredno, J., Wendland, M., Derugin, N., Ohara, P. and Wintermark, M. (2011). High and Low Molecular Weight Fluorescein Isothiocyanate (FITC)-Dextrans to Assess BloodBrain Barrier Disruption: Technical Considerations. Trans/ Stroke Res 2(1): 106-111.

14. Linden, J. R., Flores, C., Schmidt, E. F., Uzal, F. A., Michel, A. O., Valenzuela, M., Dobrow, S. and Vartanian, T. (2019). Clostridium perfringens epsilon toxin induces blood brain barrier permeability via caveolae-dependent transcytosis and requires expression of MAL. PLOS Pathog 15(11): e1008014.

15. Linden, J. R., Telesford, K., Shetty, S., Winokour, P., Haigh, S., Cahir-McFarland, E., Antognetti, G., Datta, A., Wang, T., Meier, W. and Vartanian, T. (2018). A Novel Panel of Rabbit Monoclonal 
Antibodies and Their Diverse Applications Including Inhibition of Clostridium perfringens Epsilon Toxin Oligomerization. Antibodies (Basel) 7(4): 37.

16. Machholz, E., Mulder, G., Ruiz, C., Corning, B. F. and Pritchett-Corning, K. R. (2012). Manual

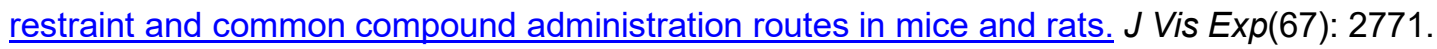

17. Nag, S. (2003). Blood-brain barrier permeability using tracers and immunohistochemistry. Methods Mol Med 89: 133-144.

18. Preston, J. E., Joan Abbott, N. and Begley, D. J. (2014). Transcytosis of macromolecules at the blood-brain barrier. Adv Pharmacol 71: 147-163.

19. Rumah, K. R., Ma, Y., Linden, J. R., Oo, M. L., Anrather, J., Schaeren-Wiemers, N., Alonso, M. A., Fischetti, V. A., McClain, M. S. and Vartanian, T. (2015). The Myelin and Lymphocyte Protein MAL Is Required for Binding and Activity of Clostridium perfringens epsilon-Toxin. PLoS Pathog 11(5): e1004896.

20. Saunders, N. R., Dziegielewska, K. M., Mollgard, K. and Habgood, M. D. (2015). Markers for blood-brain barrier integrity: how appropriate is Evans blue in the twenty-first century and what are the alternatives? Front Neurosci 9: 385.

21. Schnitzer, J. E., Oh, P., Pinney, E. and Allard, J. (1994). Filipin-sensitive caveolae-mediated transport in endothelium: reduced transcytosis, scavenger endocytosis, and capillary permeability of select macromolecules. J Cell Biol 127(5): 1217-1232.

22. Schubert, W., Frank, P. G., Razani, B., Park, D. S., Chow, C. W. and Lisanti, M. P. (2001). Caveolae-deficient endothelial cells show defects in the uptake and transport of albumin in vivo. J Biol Chem 276(52): 48619-48622.

23. Shortt, S. J., Titball, R. W. and Lindsay, C. D. (2000). An assessment of the in vitro toxicology of Clostridium perfringens type D epsilon-toxin in human and animal cells. Hum Exp Toxicol 19(2): 108-116.

24. Sultan, F. A. (2013). Dissection of different areas from mouse hippocampus. Bio Protoc 3(21).

25. Tietz, S. and Engelhardt, B. (2015). Brain barriers: Crosstalk between complex tight junctions and adherens junctions. J Cell Biol 209(4): 493-506.

26. Wolburg, H. and Lippoldt, A. (2002). Tight junctions of the blood-brain barrier: development, composition and regulation. Vascul Pharmacol 38(6): 323-337. 